\title{
Voltage and Frequency Control for Future Power Systems: the ELECTRA IRP Proposal
}

\author{
D’hulst, R.; Merino Fernandez, J.; Rikos, E.; Kolodziej, D.; Heussen, Kai; Greibel, D.; Temiz, A.; Caerts, C.
}

Published in:

Proceedings of 2015 International Symposium on Smart Electric Distribution Systems and Technologies (EDST)

Link to article, DOI:

10.1109/SEDST.2015.7315215

Publication date:

2015

Document Version

Peer reviewed version

Link back to DTU Orbit

Citation (APA):

D'hulst, R., Merino Fernandez, J., Rikos, E., Kolodziej, D., Heussen, K., Greibel, D., Temiz, A., \& Caerts, C. (2015). Voltage and Frequency Control for Future Power Systems: the ELECTRA IRP Proposal. In Proceedings of 2015 International Symposium on Smart Electric Distribution Systems and Technologies (EDST) (pp. 245 250). IEEE. https://doi.org/10.1109/SEDST.2015.7315215

\section{General rights}

Copyright and moral rights for the publications made accessible in the public portal are retained by the authors and/or other copyright owners and it is a condition of accessing publications that users recognise and abide by the legal requirements associated with these rights.

- Users may download and print one copy of any publication from the public portal for the purpose of private study or research.

- You may not further distribute the material or use it for any profit-making activity or commercial gain

- You may freely distribute the URL identifying the publication in the public portal 


\title{
Voltage and Frequency Control for Future Power Systems: the ELECTRA IRP Proposal
}

\author{
R. D’hulst*, J. Merino Fernández ${ }^{\dagger}$, E. Rikos ${ }^{\ddagger}$, D. Kolodziej ${ }^{\S}$, K. Heussen $₫$, D. Geibell ${ }^{\Perp}$ A. Temiz ${ }^{* *}$, and C. Caerts* \\ *VITO, Unit Energy Technology, Boeretang 200, 2400 Mol, Belgium \\ Email: reinhilde.dhulst@ vito.be \\ ${ }^{\dagger}$ Tecnalia, Parque Científico y Tecnológico de Bizkaia. C/Geldo, Edif. 700, Derio (Bizkaia), Spain \\ $\ddagger$ CRES, 19th km Marathonos Ave, 19009, Pikermi Attiki, Greece \\ §IEN, Institute of Power Engineering, Gdansk Division, Mikolaja Reja 27,80-870 Gdansk, Poland \\ IDTU, Center for Electric Power and Energy (CEE), Richard Petersens Plads 322, 2800 Kgs. Lyngby, Denmark \\ $\|_{\text {Fraunhofer IWES, Division Systems Engineering and Distribution Grids, Koenigstor 59, D-34119 Kassel, Germany }}$ \\ **TUBITAK, MRC Energy Institute, METU Campus Ankara, Turkey
}

\begin{abstract}
In this paper a high level functional architecture for frequency and voltage control for the future $(2030+)$ power system is presented. The proposal suggests a decomposition of the present organization of power system operation into a "web of cells". Each cell in this web is managed by a single system operator who assumes responsibility for real-time balance and voltage control of the cell, minimizing the dependency on inter-cell communication for secure system operation. The web-of-cells architecture ensures overall system stability by a combination of decentralized and distributed control patterns for frequency and voltage control. In each control cell, the operator maintains an accurate view on the overall cell state, based on adequate monitoring capabilities, and ensures secure operation by allocating and dispatching reserves located in the cell. Intercell coordination provides for efficient system-wide management and economic optimization.
\end{abstract}

\section{INTRODUCTION}

An inceasingly renewables-based and distributed energy system as expected in Europe for the time beyond 2030 requires a revision of the power system operation principles so that approapriate level of system security and resilience can be maintained. he ELECTRA Integrated Research Programme (IRP) [1] has been set-up by the European Energy Research Alliance (EERA) partners in the joint programme Smart Grids to research radical new control solutions for voltage and frequency control in the 2030 power system.

In this paper a high level functional architecture for frequency and voltage control for the future (2030+) power system is proposed. Based on a number of scenario assumptions regarding the 2030+ power system, a new control architecture to better address the fundamental changes of the future power system is presented. This work focuses on the highlevel functional control architecture related to the real-time reserves activation currently performed by transmission system operators (TSOs). Moreover, this work covers the correction of real-time imbalances -hence frequency deviations-, as well as the regulation of the grid voltage.

It is expected that due to the expected changes, further elaborated in Section II, the future frequency and voltage control can no longer be effectively managed in a TSO-centric manner exclusively. Instead, a new approach is proposed, that leverages innovative monitoring systems based on a fully instrumented network, and dynamic autonomous distributed control functions especially including distribution networks.

The focus of this work is on the development of new frequency and voltage control schemes applicable to the functional architecture developed within ELECTRA IRP. Reserves must be contracted through a market party taking into account (regulatory) requirements related to amounts, types, characteristics, location, but this procurement itself is considered out of scope for this work.

\section{Key TRends And Consequences}

According to the European Commission Energy Roadmap 2050 for long-term plans [2], by the year 2030, around 25\% of the primary energy will come from RES and the percentage will increase until up to $60 \%$ by 2050 . In this context, the ELECTRA consortium has indentified seven key trends and assumptions for future power grids:

1) Generation will shift from classical dispatchable units to intermittent renewables

Based on various reports, it is expected that by 2030, between $52 \%$ to $89 \%$ of electricity production will stem from RES [3], [4]. As a consequence, there is a paradigm shift needed from generation following load to load following generation. Also, an increased need arises for balancing reserves activations to correct in real-time the residual imbalances caused by forecast errors and variability of intermittent generation and loads. Even though on a global level these errors may partially cancel each other out, they may cause imbalances resulting in insecure power flows that may eventually cause system instability.

2) Generation will shift from central Transmission System connected generation to decentralized Distribution System connected generation 
The production share of RES connected to distribution grids will increase. As a consequence risk of local voltage problems and congestions have to be handled. Also, the location of the sources of voltage issues and balancing problems that require reserves activation, will shift from central transmission system level to distribution system level. Additionally, the resources that can help to address voltage and balancing problems, i.e. resources that can provide ancillary services support, will partly move from transmission system level to distribution system level. Therefore a central system operator requires information from operators of underlying voltage levels in order to dispatch efficiently. Finally, the distribution and availability of resources may vary significantly from location to location.

3) Generation will shift from few large units to many smaller units

Electricity production units connected to the distribution grid are typically much smaller than large central power plants. Moreover, a transition is going on within electricity production investments from an "OPEX"-driven model towards a model that is more "CAPEX"-driven, leading to more investments in smaller production units as opposed to larger (classic) production plants [5]. As a consequence, there will be more places, and chances, where incidents (such as generation outages) can happen, but each individual incidents will have a smaller, local impact. Since the production portfolio within the overall power system will be subjected to changes throughout the day (e.g. renewable generators are weather dependent), the electromechanical time constant of the power system will depend on the time of the day. Also, the replacement of large synchronous generation and loads by converter coupled generation and loads without counteractive measures will lead to reduced system inertia. This results potentially in much higher rate of change of frequency (ROCOF).

4) Electricity Consumption will increase significantly Due to the GHG emission reduction targets, there is a drive towards the electrification of transport and heating/cooling, resulting in an expected increase of the electricity consumption of around 43\% [6]. This increase will be partially compensated by the electricity consumption reduction resulting from energy efficiency measures and targets. The consequences are that the grid load will increase, increasing (the risk for) congestion and local voltage problems. This will in particular be the case in the distribution grid, where the majority of additional load resulting from the electrification of heating (domestic and tertiary sector) and transport will be situated, and where as well the distributed RES generation is located. Due to increased share heating/cooling consumption, it becomes much more temperature-dependent and thus less predictable and volatile. On the other hand, these loads represent a large potential of flexibility and storage in the grid. An increase in consumption, increases the risk for coinciding consumption peaks, in turn causing large power flows. Power peaks are expected especially if consumers will be encouraged to consume electricity following the production pattern of renewable production.

5) Electrical storage will be a cost-effective solution for offering ancillary services

According to the recommendations for a European Energy Storage Technology Development Roadmap, prices of (electrical) storage are projected to drop, making distributed storage a competitive solution compared to traditional resources for reserve services [2], [7], [8]. Storage is well suited to deal with continuous small up and down fluctuations caused by intermittency and forecast errors. Moreover, it has a larger flexibility range in both directions and fast reaction time. Additionally, storage at distribution level can provide voltage support control thanks to reactive power compensation and improve voltage quality.

6) Ubiquitous sensors will vastly increase the power systems observability

With the proliferation of distributed generation, and the price of sensors and solutions set to fall dramatically over the next few years, the inclusion of sensing and monitoring systems is starting to make more economic sense [9]. As a consequence there will be many more measurement points at all voltage levels, such as Phase Measurement Units, smart metering infrastructure, etc. providing system operators the possibility to get a holistic view on their grid.

7) Large amounts of fast reacting distributed resources (can) offer reserves capacity

Vast amounts of flexible loads will be available at all voltage levels (especially at the low voltage levels). The same holds for local storage. Both of these have very fast reaction and ramp times. Additionally, both of these will be connected through public ICT infrastructure to grid operators and market parties offering there flex-capabilities as a service.

\section{Conceptual Functional Architecture: WEB-OF-CELLS}

Based on the ELECTRA key trends as outlined in the previous section, the present grid management structure and organization for frequency and voltage control, with the TSO being responsible for reserves activation in its Control Area, is no longer effective [10]. The approach today, with the TSO as 
single, central actor responsible has proven effective because the resources for reserves needed to address frequency (or balance) issues and voltage problems, are (mainly) located centrally at the HV level. With the shift to the distribution grid of the problem causes, as well as the reserves resources that must be activated to resolve them, a new control architecture may be more appropriate. Moreover, local imbalances leading to insecure load-flows may stay unnoticed at system level, thus a new, decentralized, approach for balance control might prove to be more adequate.

In ELECTRA IRP, an architecture is proposed that goes for a decentralized managed future, where the power system is divided in grid units, called Control Cells, that provide local balancing and voltage control. In this proposal, the EU power grid is decomposed into a Web-of-Cells structure, illustrated in Fig. 1. The Control Cells are defined as:

A group of interconnected loads, distributed energy resources and storage units within well-defined grid boundaries corresponding to a physical portion of the grid and corresponding to a confined geographical area.

Note that being able to operate in island mode is not a requirement of a control cell. Each control cell has assigned a Control Cell Operator who takes responsibility for the realtime reserves activation and dispatching in his own cell (i.e. assuming responsibility similar to former TSO responsibility in its Control Area). In each control cell, the Control Cell Operator maintains an accurate view on the cell state, and dispatches reserves located in the cell in a secure manner based on his knowledge of the cell state. In principle, no global system state information is required for this. In this way, a divide and conquer way of tackling voltage and balancing issues is implemented. Moreover, local problems are resolved locally, in the cell (simple and effective control paradigm) in a fast and secure manner, limiting complexity and communication overhead (i.e. no bidirectional communication between DSO and TSO is required for reserve activation). There is no need to expose local problems at global system level. A control cell operator is responsible for the balance within his own control cell. A control cell is considered in balance when it is able to follow the (day-ahead) consumption/generation schedule. For maintaining that balance he can procure reserves from within his cell but also cross cell border reserves from neighbouring cells. Control Cells have adequate monitoring infrastructure installed, as well as local reserves capacity enabling them to resolve voltage and cell balancing problems locally (control cells are dimensioned accordingly).

While the cell-based 'solve local problems in the cell' approach is simple and effective, it has the consequence that global reserves activation optimization is disregarded. Examples of such system-wide optimizations are:

- Economic optimization, by replacing (automatically activated) restoration reserves by more cost-effective restoration reserves

- Imbalance netting, system-wide reduction of opposite sign activations

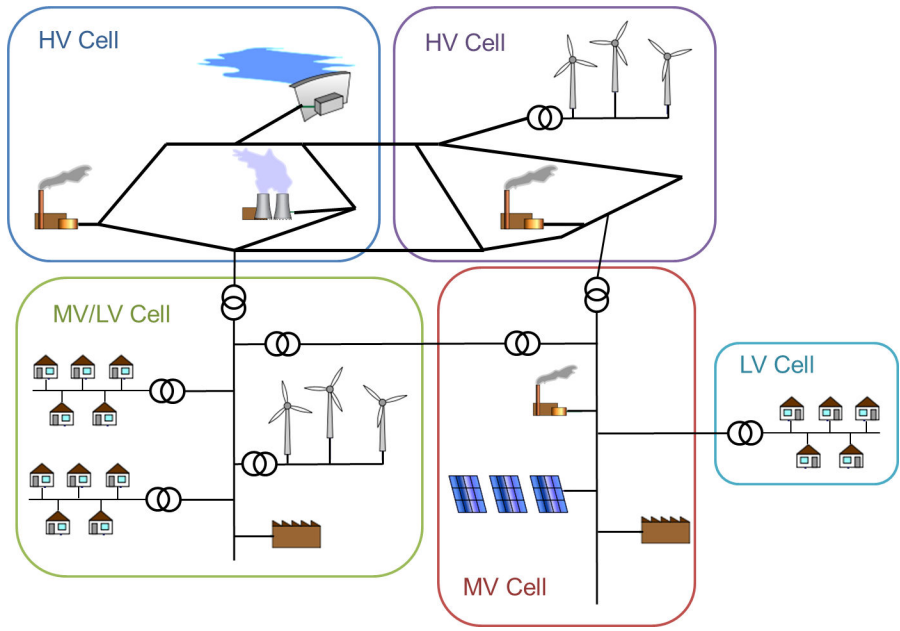

Fig. 1. Schematic illustration of proposed "Web-of-Cells" architecture

Therefore, the proposed control architecture will add an intercell coordination control layer to support system-wide optimized reserves activation if the control cell state and system state allows. It must be noted though that by allowing inter-cell coordination, the local control cell balance will not necessarily be completely restored by activating balancing reserves in an adjacent control cell. Still, on a system-wide scale system balance must be reached.

In the proposed web-of-cell based architecture, control cell operators are responsible to contribute to containing and restoring system frequency, as well as containing local voltage within secure and stable limits. For this purpose, proposals for frequency and voltage control within a web-of-cells system are developed, and explained in the following sections. It must be noted that by moving to a cell-based architecture, different observables and control aims may be required. Therefore, a sound cell-based architecture is more than the transpose of existing practices from the present TSO to a control cell operator.

\section{BALANCE CONTROL}

Frequency deviations result from active power imbalances between consumption/load/import and generation/export. Frequency stability is system wide issue. Nowadays frequency control is designed as cascaded control from fast automatic primary (containment) and secondary (restoration) control to slower manual and economically optimized tertiary control. The proposed cell-based architecture still applies the main principles of Load-Frequency Control [11], and additionally introduces a dedicated inertia control for limitation of rate of change of frequency (ROCOF). These principles are however applied at control cell level instead of at Control Area level, an overview of the proposed mechanism is shown in Fig. 2. As a result, the main control objective within each control cell is to maintain the balance within the cell, and by this indirectly restore the system frequency. Moreover, not every cell-imbalance is visible through the frequency at system level, 


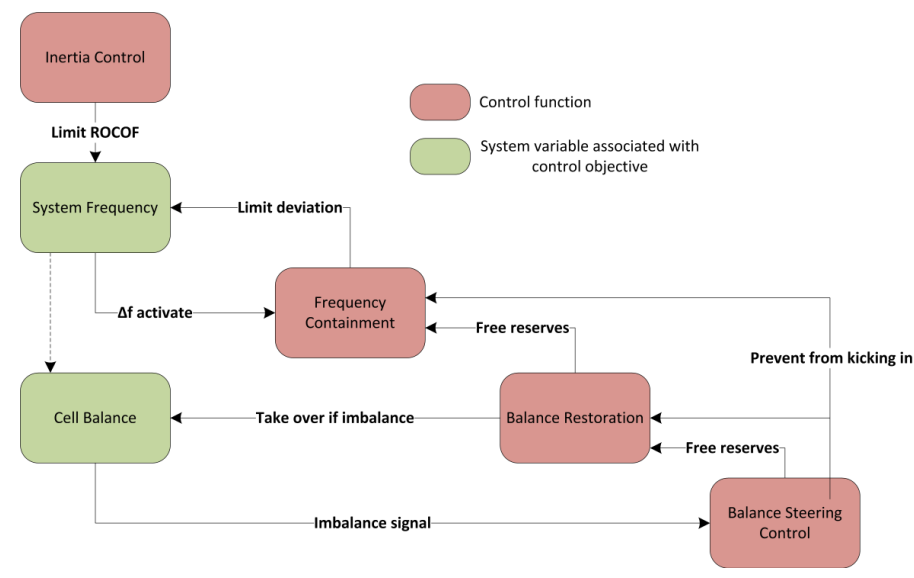

Fig. 2. Overview of proposed balance control structure of a control cell

therefore it seemed more suitable to use the term Balance Control instead of Frequency Control.

\section{A. Inertia Response Power Control}

Inertia response power is needed within the overall power system in order to keep ROCOF due to active power changes, e.g. caused by disturbances or load changes, within acceptable limits. In todays power system, the ROCOF is limited by inertial response power due to changes in the stored kinetic energy in the synchronous generators, resulting in continuous power exchanges with the grid that counteract frequency changes. However, in the future power system two challenges need to be tackled with regard to inertia response power control: (1) Converter-coupled generation increases while rotating generation decreases and (2), the generation mixes by means of the ratio between rotating and static generation will strongly vary during the day. Therefore situations could occur where direct-coupled inertia response power, i.e. from rotating machines, has to be replaced by inertia response power provided by converter-coupled units or loads. This kind of functionality should replicate the effect of inertia response power of a synchronous generating unit to a prescribed level of performance. The control objectives of inertia response power control are:

1) Limitation of ROCOF to a predefined maximum value

2) Support frequency containment control (FCC) until FCC is fully activated

The principles of the proposed control scheme are shown in Fig.3. The inertia response power control functionality of indirect or synthetic inertia could be activated/deactivated by the Control Cell Operator. Therefore the Control Cell Operator has to monitor and observe the inertia response power capability to initiate appropriate actions depending on the required inertia response power. The dimensioning of the required inertia response power should be coordinated with a.o. the Frequency Containment Control and across Control Cell boundaries. The functionality of inertia response power itself depends on local frequency and rate of change of frequency, therefore each unit/load involved in inertia response power

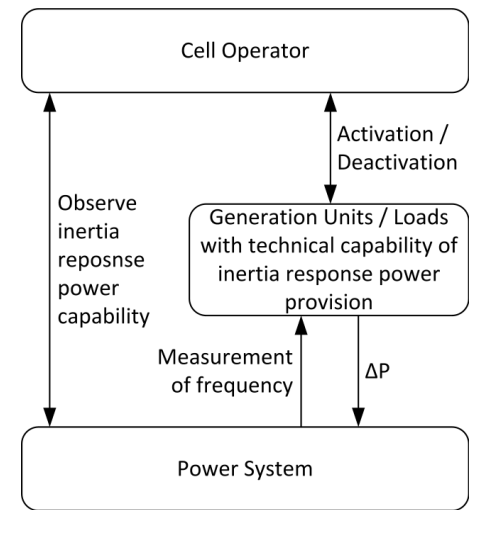

Fig. 3. Main principles of inertia control.

control automatically changes its active power contribution or consumption depending on a predefined characteristic. The basic requirement to be fulfilled is that inertia response power is proportional to the negative time derivative of frequency. It is assumed that an emulation of direct-coupled inertia is not an absolute requirement. Also, alternative characteristics could be implemented in converter-coupled units/loads which are more suitable for them if they are not contradicting with the requirements of power system control.

\section{B. Frequency Containment Control}

The goal of frequency containment control (FCC) is to stabilize frequency deviation to a set safe band. This is achieved by activating resources providing containment reserves automatically based on local frequency measurements. In case of a power imbalance the objectives are:

1) To support upstream control of inertia response power control to keep the maximum dynamic frequency deviation limit through sufficient fast activation of FCC

2) To keep the maximum steady-state frequency deviation until downstream control actions take over to restore system frequency by means of subsequent balancing mechanisms.

In todays power system FCC is predominantly provided by conventional power plants. This results from existing market rules, e.g. in Germany FCC has to be provided for a complete week and with a minimum capacity of $1 \mathrm{MW}$ power reserve. This limits the potential contribution of units with a primary source depending on the weather or with limited storage capacity. Therefore a transition to a more flexible FCC is proposed. Especially converter-coupled sources can provide due to their high dynamics and fast response times a valuable contribution. It has to be taken into account that energy reservoir of converter-coupled units as e.g. battery units are limited compared with conventional power plants. Therefore it is advisable to develop a framework where every unit (generation and load) is able to bring in its strengths based on the technology characteristics. This offers the possibility for an economic optimization through the distribution of the FCC on different kind of generators or loads. As consequence, 
the reserves will be much more distributed across the power grid (within each cell) and composed of many small contributors instead of few large contributors. Note that (especially important at low-voltage level), the activation of FCC reserves might need to take into account the local grid status, to avoid causing over- or undervoltages.

\section{Balance Restoration Control}

The goal of Balance Restoration control (BRC) is to restore control cell balance and by doing so restoring inter-cell power flows to secure values. Based on the difference between scheduled power flow and measured/actual power flow across the cell borders, also referred to as the Balance Restoration control error, Balance Restoration reserves which are available within the control cell are activated. Restoration Reserves may be offered by loads, production units as well as storage units. Response of BRC Reserves activation orders should be sufficiently fast. It is assumed that (almost) all prosumers, that are connected through public IP infrastructure, will be able to offer fast Balance Restoration reserve capacity, e.g. through their flexible loads, and possibly local storage. The combination of all those resources will give the Control Cell Operator a sufficient amount of restoration reserve capacity. By the activation of resources providing BRC reserves, containment reserves are freed up to deal with subsequent incidents. Each Control Cell Operator is responsible for activating BRC reserves when an imbalance within his cell is detected. Within the BRC control layer, it is assumed that only resources from within the control cell can be procured as Balance Restoration reserves. Dispatching the reserves by the Control Cell Operator is based on an ordered list taking into account economic factors, but potentially others as well (e.g. fairness). Before activation the local grid status is checked so that activating reserves does not cause congestion or voltage issues within the control cell.

\section{Balance Steering Control}

The objective of Balance Steering Control (BSC) is twofold:

1) Freeing up the Balance Restoration Reserves by replacing them by balance steering control reserves, possibly from other neighbouring control cells, and possibly cheaper. Also by reducing the overall, system wide, amount of BRC activations by exploiting opposite sign imbalances in other control cells (= imbalance netting). This means that the goal to restore the control cell balance (and with that : the tie connection load flows to their scheduled values) is relaxed, and that new load flow values are set as new scheduled baselines.

2) Pro-active dispatch based on short term forecasts, and thus preventing the activation of frequency containment and restoration reserves. This way, the system becomes more reliable against contingencies and its operating cost is optimized.

BSC deploys resources not only within the control cell but also from neighbouring control cells. Additional indicators such as operating costs of reserves can be used for validation of cost-optimization algorithms deployed by the balance steering

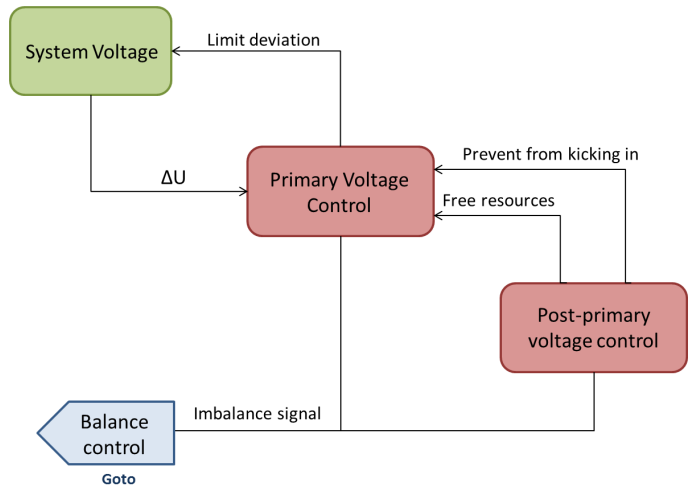

Fig. 4. Overview of proposed voltage control structure of a control cell

control. Also, similar as with BRC, before activation the local grid status is checked so that activating reserves does not cause congestion or voltage issues within the control cell or across cell borders.

\section{Voltage CONTROL}

To maintain the voltages within allowable limits, Control Cell Operators will need to procure services from units connected to its grid or from neighbouring control cells by coordination with the respective control cell operators. Voltage stability is a local issue, therefore it is appropriate to solve voltage issues using local resources from within the respective control cell. Furthermore, since it is expected that more generating units will be connected at distribution level, fewer big power plants will be available for voltage control services at transmission level. As a consequence, there will be a displacement of responsibilities from transmission to distribution level. The obligations concerning voltage control will have to be shared between control cell operators at the different voltage levels, since the traditional approach with most of the authority remaining at transmission operators will be no longer valid. Two control layers are identified within voltage control: primary voltage control and post-primary voltage control, as can be observed in Fig. 4.

\section{A. Primary Voltage Control}

The goal of Primary Voltage Control (PVC) is to act over the reactive (or active) power injection in the point of interconnection of the device. PVC is an automatic control accomplished by fast-acting devices (such as the automatic voltage controllers of the generation groups). It operates in the range of milliseconds. Based on the measured voltage at the interconnection of the device, the reactive and/or active power flow of the device is regulated so that the voltage in the node sets close to the set-point. The primary voltage control setpoint is received from the post-primary voltage control layer. Operationally, no fundamental change compared to todays primary voltage control is foreseen, except that the resources used for primary voltage control will be different: generating units (in the broadest sense) as well as loads, storage devices and FACTS. These resources will be procured 
within every control cell, and will thus be distributed over different voltage levels. As mentioned above, it is possible that, particular at LV level, the use of active power will be needed to have an adequate effect on the voltage level. One could argue that the use of active power will cause new/additional imbalances. This, however, is not problematic since we rely on the balance control to correct imbalances. After all, for balance control it does not make a difference whether an imbalance is caused by a forecast error, an incident or a voltage control action. Thus, this gives voltage control a higher priority over balance control: voltage issues are fixed before balance control makes sure that balance is restored.

\section{B. Post-Primary Voltage Control}

Post-primary voltage control (PPVC) has the commitment to bring the voltage levels in the nodes of the power system back to nominal values while optimizing the reactive power flows in order to reduce the losses in the network. In the Webof-Cells structure, PPVC is intended to replace the secondary and tertiary voltage control schemes existing in power grids by a decentralized control, located at control cell level. It is clear that mainly reactive power will be used to restore any voltage issue. The required reactive power may be delivered from generating units (of any kind) as well as storage, or any other unit capable of offering reactive power. However, if active power proves to be more effective, and optimal, to be used to control the voltage level (in particular at LV levels), active power may be procured as PPVC resource as well. Then the same comment with respect to impact on balance in PVC applies here as well: balance control is used to restore the balance. Each control cell is responsible for its own voltage control while a close coordination guarantees the provision of PPVC service between neighbouring cells. Each time a voltage issue is detected, the Control Cell Operator determines its necessary PPVC resources by taking into account technical as well as economic constraints. Before activating any PPVC resource, the Control Cell Operator determines whether the activation causes congestion issues that could put the cell stability into risk. Probably many PPVC resources will be located at MV levels, with possibility of service contributions to LV layers as well as HV layers. The PPVC mechanism assures the possibility of supplying Voltage Control resources to e.g. HV control cells if there is a lack of self-procured resources within the HV control cell.

\section{CONCLUSION}

In this paper a high level functional architecture for frequency and voltage control for the future (2030+) power system is proposed. The need for a transition towards a new architecture is based on a number of assumptions regarding the 2030+ power system. In the proposal, the EU power grid is decomposed into a Web-Of-Cells structure. Each control cell has adequate monitoring infrastructure installed, as well as local reserves capacity enabling them to (partially) resolve voltage and control cell balancing problems locally. Each control cell is managed by a single system operator, who takes responsibility for the real-time reserves activation and dispatching in his cell. Inter-cell reserve exchanges and coordination is included for optimal system-wide management. In each control cell, the control cell operator maintains an accurate view on the overall cell state, and dispatches reserves located in the cell in a secure manner based on his knowledge of the cell state. In principle, no global system state information is required for this. In the proposed web-ofcell based architecture, control cell operators are responsible to contribute to containing and restoring system frequency, as well as containing local voltage within secure and stable limits. For this purpose, proposals for balance and voltage control within a web-of-cells system are developed. In comparison to state of the art power system control the inertia control is introduced. This control assures that sufficient units (also inverter-coupled) contribute to the limitation of ROCOF. For this, system inertia is required as a new observable to select the contributing units depending on the system state. The main further development for FCC is to increase flexibility by usage of multiple kinds of units depending on their technological strengths. Frequency Restoration and Replacement is replaced by Balance Restoration and Balance Steering Control, in which the main objective is the restoration of balance within each Control Cell. The main further development for PVC is the usage of multiple kinds of units for stabilizing grid voltage. Post-Primary Voltage Control replaces secondary and tertiary voltage control, the goal is to solve voltage issues as local as possible.

\section{ACKNOWLEDGMENT}

The research leading to these results has received funding from the European Union Seventh Framework Programme (FP7/2007-2013) under grant agreement n 609687. The authors are solely responsible for the content of this publication.

\section{REFERENCES}

[1] ELECTRA IRP: European liaison on electricity committed towards long-term research activity integrated research programme. [Online]. Available: http://www.electrairp.eu/

[2] European Comission, "Energy roadmap 2050," 2012.

[3] G. Resch, C. Panzer, and A. Ortner, "2030 RES targets for Europe: a brief pre-assessment of feasibility and impact." Report, 2014.

[4] S. Samadi and S. Lechtenbhmer, "Technical analysis and comparison of underlying scenarios for the forthcoming European Commission. white paper on a 2030 climate and energy policy framework." 2014.

[5] J. Albrecht and R. Laleman, "Policy trade-offs for the belgian electricity system," Report, 2014. [Online]. Available: http://www.ceem.ugent.be/en/reports.htm

[6] European Renewable Energy Council, "RE-thinking 2050, a 100Energy Vision for the European Union," 2010.

[7] EASE/EERA, "Joint EASE/EERA recommendations for a European Energy Storage Technology Development Roadmap towards 2030," 2013.

[8] IEC, "Electrical energy storage, white paper." 2011.

[9] Navigant Research, "Utility spending on asset management and grid monitoring technology will reach nearly $\$ 50$ billion through 2030," Report, 2014.

[10] EcoGrid, "Steps toward a danish power system with 50\% wind energy, wp2: System architecture," Final Report, 2009.

[11] European Network of Transmission System Operators for Electricity (ENTSO-E), "Network code on load-frequency control and reserves," 2013. 